\title{
HARD RESCATTERING MECHANISM IN HIGH ENERGY PHOTODISINTEGRATION OF THE LIGHT NUCLEI
}

\author{
M.M. SARGSIAN \\ Department of Physics, Florida International University Miami, FL 33199
}

\begin{abstract}
We discuss the high energy photodisintegrataion of light nuclei in which the energy of the absorbed photon is equally shared between two nucleons in the target. For these reactions we investigate the model in which photon absorption by a quark in one nucleon followed by its high momentum transfer interaction with a quark of the other nucleon leads to the production of two nucleons with high relative momentum. We sum the relevant quark rescattering diagrams, and demonstrate that the scattering amplitude can be expressed as a convolution of the large angle $N N$ scattering amplitude, the hard photon-quark interaction vertex and the lowmomentum nuclear wave function. Within this model we calculate the cross sections and polarization observables of high energy $\gamma+d \rightarrow p n$ and $\gamma+{ }^{3} \mathrm{He} \rightarrow p p+n$ reactions.
\end{abstract}

\section{Introduction}

The reactions of high energy photodisintegration of light nuclei in which the energy of the incoming photon is equally shared by two outgoing nucleons is unique in a sense that it deposits to the nuclear system a large amount of energy which should be shared at least by two nucleons in the nuclei (see e.g. ${ }^{1,2}$ ). When this energy exceeds the typical meson mass (sevearl hundred $\mathrm{MeV}$ ) one expects that the interaction picture based on a meson exchange currents should break down. In this limit the hard contribution is expected to dominate. As a result one expects that the quark gluon picture of interaction will become a relevant framework for description of the reaction.

The experiments on high energy two-body photodisintegration of the deuteron $^{3,4}$ demonstrated that starting at $\mathrm{E}_{\gamma} \geq 1 \mathrm{GeV}$ the conventional mesonic picture of nuclear interactions is indeed breaking down. One of the first predictions for $\gamma d \rightarrow p n$ reactions within QCD was based on the quark counting rule, which predicted $d \sigma / d t \sim s^{-11}$. This prediction was based on the hypothesis that the Fock state with the minimal number of partonic constituents will dominate in two-body large angle hard collisions ${ }^{5}$. Although successful in describing energy dependences of number of hard 
processes, this hypothesis does not allow to make calculation of the absolute values of the cross sections. Especially for reactions involving baryons, the calculations within perturbative QCD underestimate the measured cross sections by orders of magnitude see e.g. ${ }^{6}$. This may be an indication that in the accessible range of energies bulk of the interaction is in the domain of the nonperturbative $\mathrm{QCD}^{6,7}$, for which the theoretical methods of calculations are very limited.

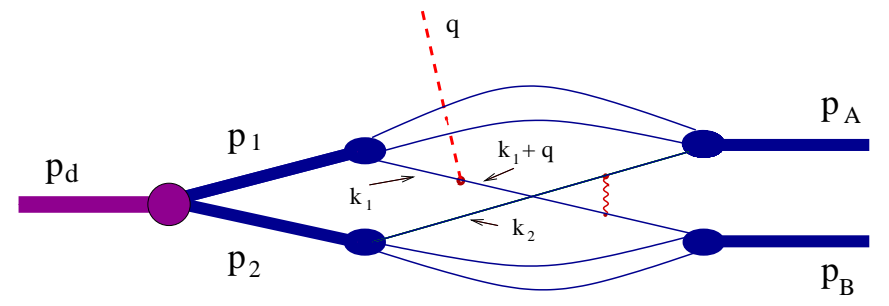

Figure 1. Quark Rescattering diagram.

\section{Hard Rescattering Model}

Recently we suggested a model in which the absorption of the photon by a quark of one nucleon, followed by a high-momentum transfer (hard) rescattering with a quark from the second nucleon, produces the final two nucleon state of large relative momenta. The typical diagram representing such a scenario is presented in Figure 1. Based on the analysis of these type of diagrams we find that:

- the dominant contribution comes from the soft vertices of $d \rightarrow N N$ transition, while quark rescattering proceeds trough hard gluon exchange

- the $d \rightarrow N N$ transition can be evaluated based on the conventional deuteron wave function calculated using the realistic nucleonnucleon potentials.

- the structure of hard interaction for the rescattering part of the reaction is similar to that of hard NN scattering.

- as a result the sum of the multitude of diagrams with incalculable nonperturbative parts of the interaction can be expressed through the experimentally measured amplitude of hard $N N$ scattering.

\section{Kinematics and the Cross Section}

We consider the kinematics of sufficiently large energies $E_{\gamma} \geq 2 \mathrm{GeV}$ and momentum transfer $-t,-u \geq 2 G e V^{2}$. These restrictions allow to make 
a two important approximations: first is that the mass of the intermediate hadronic state produced in the $\gamma N$ interaction is in deep inelastic continuum, thus the partonic picture is relevant. The second one is that the quark rescattering is hard and can be factorized from the soft nuclear wave function. We evaluate Feynman diagrams such as Fig. 1, in which quarks are exchanged between nucleons via the exchange of a gluon. All other quark-interactions are included in the partonic wave function of the nucleon, $\psi_{N}$. We use a simplified notation in which only the momenta of the interacting quarks require labeling. The scattering amplitude $T$ for photo-disintegration of the deuteron (of four-momentum $p_{d}$ and mass $M_{d}$ ) into two nucleons of momentum $p_{A}$ and $p_{B}$ is given by:

$$
\begin{aligned}
& T=-\sum_{e_{q}} \int\left(\frac{\psi_{N}^{\dagger}\left(x_{2}^{\prime}, p_{B \perp}, k_{2 \perp}\right)}{x_{2}^{\prime}} \bar{u}\left(p_{B}-p_{2}+k_{2}\right)\left[-i g T_{c}^{F} \gamma^{\nu}\right]\right. \\
& \left.\frac{u\left(k_{1}+q\right) \bar{u}\left(k_{1}+q\right)}{\left(k_{1}+q\right)^{2}-m_{q}^{2}+i \epsilon}\left[-i e_{q} \epsilon^{\perp} \cdot \gamma^{\perp}\right] u\left(k_{1}\right) \frac{\psi_{N}\left(x_{1}, p_{1 \perp}, k_{1 \perp}\right)}{x_{1}}\right) \\
& \left\{\frac{\psi_{N}^{\dagger}\left(x_{1}^{\prime}, p_{A \perp}, k_{1 \perp}\right)}{x_{1}^{\prime}} \bar{u}\left(p_{A}-p_{1}+k_{1}\right)\left[-i g T_{c}^{F} \gamma_{\mu}\right] u\left(k_{2}\right) \frac{\psi_{N}\left(x_{2}, p_{2 \perp}, k_{2 \perp}\right)}{x_{2}}\right\} \\
& G^{\mu \nu} \frac{\Psi_{d}\left(\alpha, p_{\perp}\right)}{1-\alpha} \frac{d x_{1}}{1-x_{1}} \frac{d^{2} k_{1 \perp}}{2(2 \pi)^{3}} \frac{d x_{2}}{1-x_{2}} \frac{d^{2} k_{2 \perp}}{2(2 \pi)^{3}} \frac{d \alpha}{\alpha} \frac{d^{2} p_{\perp}}{2(2 \pi)^{3}},
\end{aligned}
$$

where $p_{1}$ and $p_{2}$ are the momenta of the nucleons in the deuteron, with $\alpha \equiv \frac{p_{1+}}{p_{d+}}, p_{2}=p_{d}-p_{1}$ and $p_{1 \perp}=-p_{2 \perp} \equiv p_{\perp}$. Each nucleon consists of one active quark of momenta $k_{1}$ and $k_{2}: x_{i} \equiv \frac{k_{i+}}{p_{i+}}=\frac{k_{i+}}{\alpha p_{d+}}(i=1,2)$. $G^{\mu \nu}$ describes the gluon exchange between interchanged quarks. We use the reference frame where $p_{d}=\left(p_{d 0}, p_{d z}, p_{\perp}\right) \equiv\left(\frac{\sqrt{s^{\prime}}}{2}+\frac{M_{d}^{2}}{2 \sqrt{s^{\prime}}}, \frac{\sqrt{s^{\prime}}}{2}-\frac{M_{d}^{2}}{2 \sqrt{s^{\prime}}}, 0\right)$, with $s=\left(q+p_{d}\right)^{2}, s^{\prime} \equiv s-M_{D}^{2}$, and the photon four-momentum is $q=$ $\left(\frac{\sqrt{s^{\prime}}}{2},-\frac{\sqrt{s^{\prime}}}{2}, 0\right)$.

We first observe that the denominator of the knocked-out quark propagator, when recoil quark-gluon system with mass $m_{R}$ is on mass shell, has a pole at $\alpha_{c} \equiv \frac{x_{1} m_{R}^{2}+k_{1 \perp}^{2}}{\left(1-x_{1}\right) x_{1}}$ :

$$
\left(k_{1}+q\right)^{2}-m_{q}^{2}+i \epsilon \approx x_{1} s^{\prime}\left(\alpha-\alpha_{c}+i \epsilon\right),
$$

where $\tilde{s} \equiv s^{\prime}\left(1+\frac{M_{d}^{2}}{s^{\prime}}\right)$. Next we calculate the photon-quark hard scattering vertex and integrate over the $\alpha$ using only the pole contribution in Eq.(2). Note that the dominant contribution arises from the soft component of the deuteron when $\alpha_{c}=\frac{1}{2}$, which requires $k_{1 \perp}^{2} \approx \frac{\left(1-x_{1}\right) x_{1} \tilde{s}}{2}$.

Summing over the struck quark contributions from photon scattering off neutron and proton one can express the scattering amplitudes through 
the $p n$ hard scattering amplitude within the quark interchange mecha$\operatorname{nism}(\mathrm{QIM})-A_{p n}^{Q I M}\left(s, l^{2}\right)$ as follows:

$$
T \approx \frac{i e\left(\epsilon^{+}+\epsilon^{-}\right)\left(e_{u}+e_{d}\right)}{2 \sqrt{s^{\prime}}} \int f\left(\frac{l^{2}}{s}\right) A_{p n}^{Q I M}\left(s, l^{2}\right) \Psi_{d}\left(\frac{1}{2}, p_{\perp}\right) \frac{d^{2} p_{\perp}}{(2 \pi)^{2}}
$$

where $l=p_{p}-p_{1}, \epsilon^{ \pm}=\frac{1}{\sqrt{2}}\left(\epsilon_{x} \pm i \epsilon_{y}\right)$ and $e_{u}$ and $e_{d}$ are the electric charges of $u$ and $d$ quarks. The factor $f\left(l^{2} / s\right)$ accounts for the difference between the hard propagators in our process and those occurring in wide angle $p n$ scattering. Within the Feynman mechanism ${ }^{8}$, the interacting quark carries the whole momentum of the nucleon $\left(x_{1} \rightarrow 1\right)$, thus $f\left(l^{2} / s\right)=1$. Within the minimal Fock state approximation, $f\left(l^{2} / s\right)$ is the scaling function of the $\theta_{c m}$ only with $f\left(\theta_{c m}=90^{0}\right) \approx 1^{9}$.

We compute the differential cross section averaging $|T|^{2}$ over the spins of initial photon and deuteron and summing over the spins of the final nucleons. Then we use the observation that the quark interchange topologies are the dominant for fixed angle, $\theta_{c m}=90^{\circ}$ high momentum transfer (non strange) baryon-baryon scattering. Thus in the region of $\theta_{c m} \approx 90^{\circ}$ we replace $A_{p n}^{Q I N}$ by the experimental data - $A_{p n}^{\operatorname{Exp}}$ and obtain ${ }^{9 \mathrm{a}}$ :

$$
\begin{gathered}
\frac{d \sigma^{\gamma d \rightarrow p n}}{d t}=\frac{8 \alpha}{9} \pi^{4} \cdot \frac{1}{s^{\prime}} C\left(\frac{\tilde{t}}{s}\right) \frac{d \sigma^{p n \rightarrow p n}(s, \tilde{t})}{d t} \\
\times\left|\int \Psi_{d}^{N R}\left(p_{z}=0, p_{\perp}\right) \sqrt{m_{N}} \frac{d^{2} p_{\perp}}{(2 \pi)^{2}}\right|^{2},
\end{gathered}
$$

where $\tilde{t}=\left(p_{B}-p_{d} / 2\right)^{2}$. Here $\Psi_{d}^{N R}$ is the nonrelativistic deuteron wave function which can be calculated using realistic NN interaction potentials. The function $C\left(\frac{\tilde{t}}{s}\right) \approx f^{2}(\tilde{t} / s) \approx 1$ at $\theta_{c m} \sim 90^{0}$ and is slowly varying function of $\theta_{c m}$.

Within the hard rescattering model we can calculate also the high energy photodisintegration of ${ }^{3} \mathrm{He}$ in the specially chosen kinematics, in which the absorbed photon's energy is equally shared between the two outgoing protons while the final neutron is very slow $p_{n} \leq 100 \mathrm{MeV} / \mathrm{c}$. In this case the cross section of the reaction is expressed through the nonrelativistic wave function of the ${ }^{3} \mathrm{He}$ and the amplitude of hard $p p$ scattering, with small corrections coming from soft $p n$ rescattering.

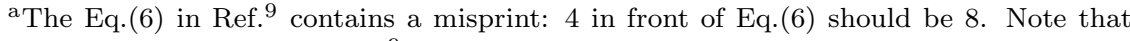
the numerical calculations in ${ }^{9}$ are done with the correct factor. 

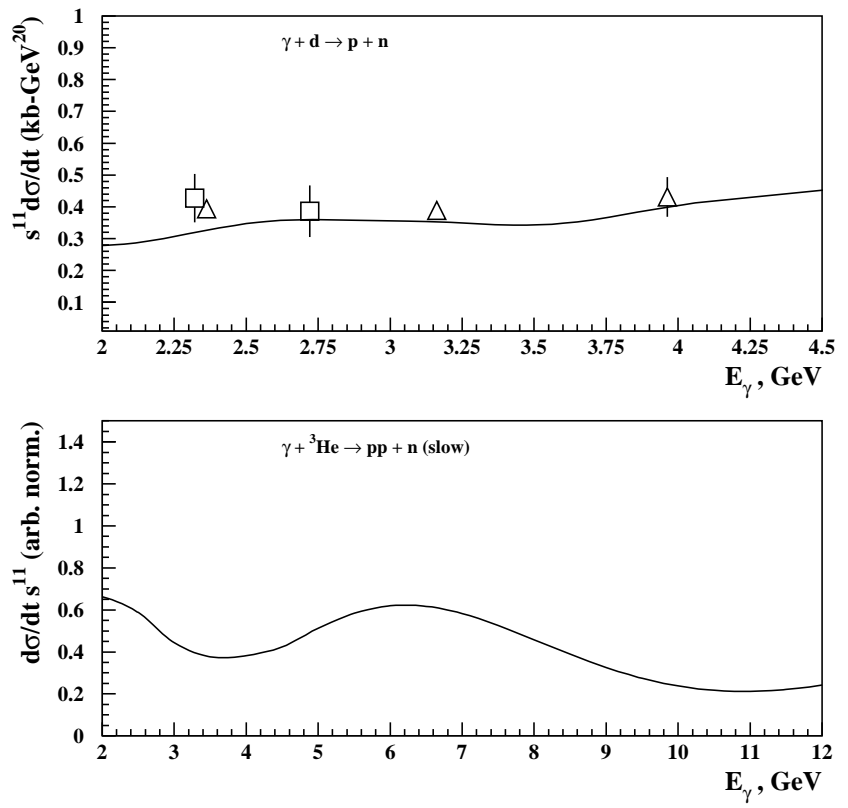

Figure 2. The $d \sigma / d t s^{11}$ as a function of $E_{\gamma}$ at $\theta_{c m}=90^{0}$. Data are from [3] (triangles) and [4] (squares).

\section{Comparison with the Data}

\subsection{Energy dependence}

One can estimate the energy dependences of the differential cross sections of the $\gamma d \rightarrow p n$ and $\gamma^{3} \mathrm{He} \rightarrow p p+n$ reactions at $\theta_{c m}=90^{0}$ using the experimental data on hard $p n$ and $p p$ scattering. Figure 2 represents the calculations and the comparison with existing data on deuteron target. Our calculation of $\gamma d \rightarrow p n$ has $\approx 25 \%$ accuracy because of the rather large experimental errors in high momentum transfer $p n$ scattering cross section. The prediction for $\gamma^{3} \mathrm{He} \rightarrow p p+n$ cross section (which should be considered preliminary) demonstrates the oscillation which is related to the observed oscillations in high momentum transfer $p p$ cross section ${ }^{10}$.

\subsection{Angular Dependence}

As we mentioned above, the hard rescattering mechanism can predict the value of $C(t / s) \approx 1$ only at $\theta_{c m}=90^{2}$. One expects that this function 
should be a smooth function of $\theta_{\mathrm{cm}}$. As a result the angular dependence of photodisintegration reaction should in general reflect the angular dependence of hard $N N$ scattering cross section.

In particular it is interesting to observe that the $p n$ scattering cross section exhibits a strong angular asymmetry with the cross sections being dominate at forward angles. As Figure 3 demonstrates, within hard rescattering model this feature is reflected also in the angular dependence of $\gamma d \rightarrow p n$ cross section. This result is in a qualitative agreement with the preliminary JLab data.

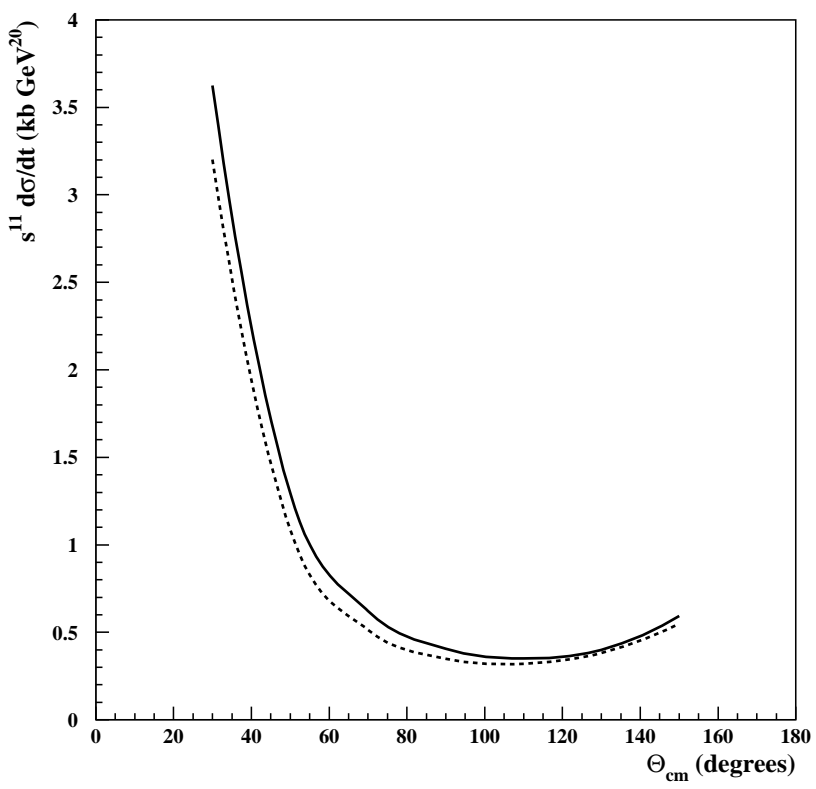

Figure 3. Angular dependence, for $\gamma d \rightarrow p n$ reaction at $E_{\gamma}=2 G e V$ (dashed curve) and $3 \mathrm{GeV}$ (solid curve).

\section{Polarization Observables}

Hard rescattering model allows also to calculate the different polarization observables of the photodisintegration reaction. Writing down explicitly the helicity indexes of interacting particles, for the scattering amplitude of Eq.(3) one obtains:

$$
T^{h, m, \eta_{2}, \eta_{1}} \approx
$$




$$
\frac{i\left(e_{u}+e_{d}\right)}{2 \sqrt{s^{\prime}}} \int f\left(\frac{l^{2}}{s}\right)<\eta_{1}, \eta_{2}\left|A_{p n}\left(s, l^{2}\right)\right| h, \lambda_{2}>\Psi_{d}^{m, h, \lambda 2}\left(\frac{1}{2}, p_{\perp}\right) \frac{d^{2} p_{\perp}}{(2 \pi)^{2}} .
$$

where $h, m, \eta_{2}$ and $\eta_{1}$ represent the helicities of incoming photon, target deuteron and two outgoing nucleons respectively. In derivation of Eq.(6) it is assumed additionally that the quark which interacts with the photon carries the total helicity of the parent nucleon. The helicity amplitudes, $A_{p n}$ in Eq.(6) can be expressed through the five independent helicity amplitudes:

$$
\begin{array}{ll}
\phi_{1}(s, t)=<++|A|++> & \phi_{2}(s, t)=<++|A|--> \\
\phi_{3}(s, t)=<+-|A|+-> & \phi_{4}(s, t)=<+-|A|-+> \\
& \phi_{5}(s, t)=<++|A|+->
\end{array}
$$

for which the following hierarchy relation can be stated in a model independent way $^{11}$ :

$$
\phi_{2}<\phi_{5}<\phi_{1}, \phi_{3}, \phi_{4}
$$

Using the definitions of Eq.(6) one can calculate the different asymmetries in the deuteron photodisintegration reaction. We are particularly interested in the recoil transverse $P_{y}$ and longitudinal $C_{x}, C_{y}$ polarizations for which the high energy data are becoming available from Jefferson Lab ${ }^{12}$. Based on Eq.(6) the predictions for these observables are as follows:

$$
\begin{aligned}
& P_{y}=-2 \operatorname{Im}\left\{\left[2\left(\phi_{1}(s, \tilde{t})+\phi_{2}(s, \tilde{t})\right)+\phi_{3}(s, \tilde{t})-\phi_{4}(s, \tilde{t})\right] \phi_{5}^{\dagger}(s, \tilde{t})\right\} / F(s, \tilde{t}) \\
& C_{x}=+2 \operatorname{Re}\left\{\left[2\left(\phi_{1}(s, \tilde{t})-\phi_{2}(s, \tilde{t})\right)+\phi_{3}(s, \tilde{t})+\phi_{4}(s, \tilde{t})\right] \phi_{5}^{\dagger}(s, \tilde{t})\right\} / F(s, \tilde{t}) \\
& C_{z}=0
\end{aligned}
$$

where $F(s, \tilde{t})=2\left|\phi_{1}(s, \tilde{t})\right|^{2}+2\left|\phi_{2}(s, \tilde{t})\right|^{2}+\left|\phi_{3}(s, \tilde{t})\right|^{2}+4\left|\phi_{4}(s, \tilde{t})\right|^{2}+$ $6\left|\phi_{5}(s, \tilde{t})\right|^{2}$. In these derivations we neglected by the $D$ wave contribution of the deuteron wave function, which is justified on the basis that the nucleon momenta which enter in the deuteron wave function in the integral of Eq.(6) is restricted, $(\leq 300 \mathrm{MeV} / \mathrm{c})$.

Based on the relation of Eq.(7) and the fact that for the on-shell amplitude $\phi_{5}=0$ at $\theta_{c m}=90^{\circ}$ one can conclude in a rather model independent way that the $P_{y}$ and $C_{x}$ should be small at $\theta_{c m}=90^{\circ}$ at $E_{\gamma} \geq 2 \mathrm{GeV}$. This result seems in qualitative agreement with the available data and the planned experiment at Jefferson Lab in $E_{\gamma} \geq 2 \mathrm{GeV}$ region will allow check these predictions in more detail.

It is interesting to note that the smallness of the polarization observables predicted in the model is not related to the commonly used assumption of 
the applicability of pQCD and related only to the fact that the considered asymmetries are determined by the small helicity component of $N N$ amplitude, $\phi_{5}$.

\section{Summary and Outlook}

The underlying hypothesis of hard rescattering model is that the dynamics of the photoproduction reaction is determined by the physics of highmomentum transfer contained in the hard scattering NN amplitude. As a result the short-distance aspect of the deuteron wave function is not important, which allows us to use the conventional deuteron wave function in numerical calculations. This hypothesis, if confirmed by additional studies, may suggest the existence of new type of calculable hard nuclear reactions in which the sum of the "infinite" number of quark interactions could be replaced by the hard amplitude of $N N$ interaction.

The prediction of the model agrees reasonably well with the existing data on high energy photodisintegration of the deuteron at $\theta_{c m}=90^{\circ}$. More data, especially with a two energetic proton final state in $\gamma+{ }^{3} \mathrm{He} \rightarrow p p$ (high $\left.p_{t}\right)+\mathrm{n}\left(p_{t} \approx 0\right)$ reaction and a more detailed angular distribution would definitely allow to verify this hypothesis. The polarization measurement also will be crucial. It is very important to extend them in the energy region where anomalies are observed in the polarized hard $p p$ scattering.

\section{References}

1. S.J. Brodsky and B.T. Chertok, Phys. Rev. Lett. 37, 269 (1976).

2. R.J. Holt, Phys Rev. C41, 2400 (1990).

3. C. Bochna et al., Phys. Rev. Lett. 81, 4576 (1998).

4. J.E. Belz et al., Phys. Rev. Lett. 74, 646 (1995).

5. S.J. Brodsky and G.R. Farrar, Phys. Rev. Lett. 31, 1153; V. Matveev, R.M. Muradyan and A.N. Tavkhelidze, Lett. Nuovo Cimento 7, 719 (1973).

6. N. Isgur and C.H. Llewellyn Smith, Phys. Rev. Lett. 52, (1984) 1080.

7. A. Radyushkin, Acta Phys. Pol. B15, 403 (1984).

8. R. Feynman, Photon Hadron Interactions, W.A. Benjamin Inc., 1972.

9. L.L. Frankfurt, G.A. Miller, M.M. Sargsian and M.I. Strikman, Phys. Rev. Lett. 84, 3045 (2000).

10. J.P. Ralston and B. Pire, Phys. Rev. Lett. 611823 (1988).

11. G. P. Ramsey and D. W. Sivers, Phys. Rev. D 45, 79 (1992).

12. K. Wijesooriya et al. [Jefferson Lab Hall A Collaboration], Phys. Rev. Lett. 86, 2975 (2001). 